\title{
Superconducting Order Parameter in Bi-Layer Cuprates: Occurrence of $\pi$ Phase Shifts in Corner Junctions
}

\author{
D. Z. Liu, K. Levin, and J. Maly \\ James Franck Institute, University of Chicago, Chicago, IL 60637
}

(Submitted to Physical Review B on 19 December 1994)

\begin{abstract}
We study the order parameter symmetry in bi-layer cuprates such as $\mathrm{YBaCuO}$, where interesting $\pi$ phase shifts have been observed in Josephson junctions. Taking models which represent the measured spin fluctuation spectra of this cuprate, as well as more general models of Coulomb correlation effects, we classify the allowed symmetries and determine their associated physical properties. $\pi$ phase shifts are shown to be a general consequence of repulsive interactions, independent of whether a magnetic mechanism is operative. While it is known to occur in d-states, this behavior can also be associated with (orthorhombic) s-symmetry when the two sub-band gaps have opposite phase. Implications for the magnitude of $T_{c}$ are discussed.

PACS numbers: 74.72. -h , 74.20.Mn, 74.50.+r, 74.62. -c
\end{abstract}

The observation in YBCO of unusual Josephson junction behavion 14 is one of the most important experimental results to emerge from the cuprate literature in recent years. Here in a Josephson SQUID experiment the two junctions are configured so that their normals lie along the $\mathrm{a}$ and $\mathrm{b}$ axes of the $\mathrm{CuO}_{2}$ plane. This measurement has been widely interpreted as support for a d-symmetry of the order parameter, as well as for a magnetic mechanism for superconductivity. In this paper we show that both of these inferences may be inappropriate. For notational precision, throughout this paper we use the terms s- ( or d- ) symmetry to correspond to states which have the same (or opposite) sign under a $\pi / 2$ rotation. Thus the d-states under consideration are not necessarily of the specific $d_{x^{2}-y^{2}}$ form.

The gap equation for bi-layer systems has been studied earlier in the cifntext of a magnetic mechanism for superconductivity d-symmetric state of the single layer problem, is transformed to a pair of in-phase d states on each of the two sub-bands, and that these compete with a pair of outof-phase s-states. Here we take the problem to a greater level of generality, establishing that this situation persists for a wide class of repulsive interactions, which are unrelated to the antiferromagnetic spin fluctuation picture. Alternate classes of the order parameter symmetry are also generated. These correspond to in-phase s-states and out-of-phase d-states. We establish how the relative stability of the two competing states is affected by changes in bandstructure, orthorhombicity, and hole filling.

It should not be surprising that d-states have a more general origin beyond the antiferromagnetic spin exchange models. In a one layer cuprate, the lattice symmetry requires that all gap states are either even (s-) or odd (d-) under a $\pi / 2$ rotation. In bi-layer systems, these one layer states generalize naturally to a pair of even or odd, in-phase or out-of-phase states, associated with each of the two sub-bands. Thus, as one of two allowed states, d-symmetry should be widespread, and independent of the microscopic details of the model.

In the presence of both intra- and inter-layer interactions $\left(V_{\|}\right.$and $\left.V_{\perp}\right)$, the weak coupling BCS gap equation becomes a set of coupled equations for the gaps on each of the sub-bands. It is simpler to write the gap equations in terms of the two sub-band gaps $\Delta_{+}, \Delta_{-}$rather than the intra-layer $\left(\Delta_{\|}\right)$and inter-layer $\left(\Delta_{\perp}\right)$ components. These are related via the unitary transformation which diagonalizes the Hamiltonian. In this model $t_{\perp}$ is the matrix element for hopping between layers; $t$ and $t^{\prime}$ refer to the first and second nearest neighbor in-plane hopping which may contain orthorhombic effects. On site Coulomb effects $U$ are assumed to enter via a renormalization of the bandstructure parameters as shown by $\mathrm{Si}$ et atd.

Following the usual procedure 8 , the gap equations become

$$
\begin{aligned}
\Delta_{+}+\Delta_{-}= & -\sum_{\mathbf{q}^{\prime}} \frac{V_{\|} \Delta_{+}}{2 E_{+}} \tanh \left(\frac{E_{+}}{2 T}\right) \\
& -\sum_{\mathbf{q}^{\prime}} \frac{V_{\|} \Delta_{-}}{2 E_{-}} \tanh \left(\frac{E_{-}}{2 T}\right) \\
\Delta_{+}-\Delta_{-}= & -\sum_{\mathbf{q}^{\prime}} \frac{V_{\perp} \Delta_{+}}{2 E_{+}} \tanh \left(\frac{E_{+}}{2 T}\right) \\
& +\sum_{\mathbf{q}^{\prime}} \frac{V_{\perp} \Delta_{-}}{2 E_{-}} \tanh \left(\frac{E_{-}}{2 T}\right)
\end{aligned}
$$

where the superconducting quasi-particle energies are $E_{ \pm}=\sqrt{\epsilon_{ \pm}^{2}+\Delta_{ \pm}^{2}}$, where

$$
\begin{aligned}
\epsilon_{ \pm}=- & 2 t\left[\cos \left(q_{x} a\right)+\cos \left(q_{y} b\right)\right] \\
& +4 t^{\prime} \cos \left(q_{x} a\right) \cos \left(q_{y} b\right) \pm t_{\perp}
\end{aligned}
$$

It follows that each of the two sub-band gaps can be written in terms of the parallel and perpendicular components as

$$
\Delta_{\|}=\left(\Delta_{+}+\Delta_{-}\right) / 2, \Delta_{\perp}=\left(\Delta_{+}-\Delta_{-}\right) / 2
$$


In the case of a magnetic pairing mechanism, the two interactions are related to components of the dynamical spin susceptibility. This susceptibility has been calculated for the bi-layer cuprate YBCOld, in the strong $U$ limit. For realistic Fermi surface shapes, and moderate in-plane and out of plane exchange interactions, the results are in reasonable agreement with neutron experiment 10. Because the in-plane magnetism is not independent of inter-plane effects any proper treatment of spin fluctuation induced superconductivity should incorporate both components. A reasonable approximation of both the theoretical results 9 and the experimental neutron data 10 is to take

$$
\begin{aligned}
V_{\|} & =g_{\|}^{2} \chi_{\|}\left(\mathbf{q}-\mathbf{q}^{\prime}\right) \\
V_{\perp} & =g_{\perp}^{2} \chi_{\perp}\left(\mathbf{q}-\mathbf{q}^{\prime}\right) \\
& =-g_{\perp}^{2} \chi_{\|}\left(\mathbf{q}-\mathbf{q}^{\prime}\right)
\end{aligned}
$$

with

$$
\chi_{\|}\left(k_{x}, k_{y}\right)=\frac{1}{\left[1+J_{o}\left(\cos k_{x} a+\cos k_{y} b\right)\right]^{2}}
$$

and $J_{o} \approx 0.3$. Here we have absorbed overall coefficients into the coupling constant prefactors $g_{\|}$and $g_{\perp}$. This model is similar to that used in Ref.5, except that we have assumed an arbitrary relation between the magnitudes of two superconducting coupling constants, which are taken to be the same in Ref.5. Moreover, we fit the dynamical susceptibility to neutron, rather than NMR data11.

It may be ngted from Eq.(4d) (as well as experimental neutron data 10 ) that the antiferromagnetic fluctuations show up as a weak peak around $(\pi / a, \pi / a)$ with short coherence lengths, suggesting that the system is far from any real instability; thus high $T_{c}$ is difficult to explain 11. Consequently we explore more general mechanisms by extending Eqs. (4a) and (4b) to the case where the overall signs are unconstrained and the $\mathbf{q}-\mathbf{q}^{\prime}$ peaks occur at arbitrary wave-vector with arbitrary peak width $J_{o}$. We define

$$
V_{\|, \perp}=\frac{\lambda_{A}, \lambda_{B}}{\left[1-J_{o}\left(\cos \left(k_{x} \pm Q_{x}\right)+\cos \left(k_{y} \pm Q_{y}\right)\right)\right]^{2}}
$$

where all signs are summed over. We divide our numerical analysis into four distinct cases in which either the inter-layer or the intra-layer interaction dominates and in which the respective interaction is repulsive $(\lambda>0)$ or attractive $(\lambda<0)$. The model is viewed as a general representation of pairing mechanisms of the electronic and phononic variety. Since the former usually derives from a generalized susceptiblity, and the latter from a phonon propagator,there are no sign changes as a function of momentum transfer. This poses important constraints on the allowed superconducting states. Our results 12 are summarized in Figures 1 and 2 for the case of characteristic wave-vectors $\mathbf{q}-\mathbf{q}^{\prime}$ along $(\pi / a, \pi / a)$ and along $(\pi / a, \pi / 2 a)$. The latter wave-vector illustrates the behavior away from the antiferromagnetic instability model, in order to show the generality of our results. Plotted in these figures are the form of the gap functions in the two regimes. The figures on the left (right) in each box correspond to intra-layer (inter-layer) dominated behavior. Our conclusions from both Figures 1 and 2 may be succinctly summarized. We find that d-symmetry is associated with repulsive and s-symmetry with attractive interactions. In phase gap behavior occurs when the intra-layer interaction is the larger; out of phase behavior arises in the opposite case. This phase dependence can be deduced from Eqs.(3). In the case of dominant interlayer effects, $\left|\Delta_{\perp}\right|>\left|\Delta_{\|}\right|$. This will occur when $\Delta_{+}$and $\Delta_{-}$have opposite signs.

To establish the generality of these results, we have varied the Fermi surface shape ( via the ratio of $t^{\prime} / t$ ), the position of the Fermi energy or hole filling and the width $J_{o}$ of the peak structure. These variations introduce only quantitative but not qualitative changes in the physical picture shown in the two figures.

It is important to note from Figs.11(a)-(c) and 2(a)-(c) that the out of phase s- and d-wave states willexhibit $\pi$ phase shifts in a corner SQUID experiment 1 . This corresponds to a change in sign of the "sum " order parameter $\Delta_{\|}$of Eqs.(3) upon varying from 0 to $\pi / 2$. While not a general feature of all solutions, its presence requires (a modest amount of ) orthorhombicity. The observation of $\pi$ phase shifts in bi-layer cuprates is thus not as strong a constraint on the order parameter symmetry as in one layer materials. All orthorhombic states which exhibit these $\pi$ phase shifts will also show finite c-axis tunneling in untwinned crystals 13 . However, twinning effects (if they average fully over the a and b-axes), will lead to a cancelation of Josephson coupling, whenever the corner SQUID experiment has the observed $\pi$ phase shift.

It should be stressed that the out of phase s-states have the additional advantage as a candidate gap symmetry, over d-states (in or out of phase), of being relatively insensitive to impurity effects. In addition this state can be compatible with neutron experiments1 10 , which show no nodal signature. In contrast to experiment, because nodes are not present, at least in the clean limit, power laws in thermodynamical properties 14 are not expected.

We have searched for nodal behavior in these s-wave states with seme care, since there is recent photoemission evidence 15 to suggest that they may exist in bi-layer BISCO. Several observations are important to note in this context. (1) As the inter-layer hopping $t_{\perp}$ becomes small the magnitudes of the two gaps become equal and they are less able to respond to orthorhomicity by producing gap anisotropy. Consequently BISCO 2212, which is believed to have a very small $t_{\perp}$, would be unlikely to exhibit nodal s-wave behavior. (2) The eight node s-state which has been conjectured as a candidate for BISCO 15 appears quite generally as a meta-stable state whose solution has a lower $T_{c}$ than the nodeless s-wave or (four node) d-wave symmetric gap. Within the manifold of meta-stable states, the more nodes, the 
FIG. 1. Superconducting gap for interactions peaked at $\mathbf{q}-\mathbf{q}^{\prime}=(\pi, \pi)$, for the case of attractive and repulsive intra-layer $\left(\lambda_{A}\right)$ and inter-layer $\left(\lambda_{B}\right)$ interactions. Figures on left (right) are for intra- (inter-) layer dominated regimes.

FIG. 2. Superconducting gap for interactions peaked at $\mathbf{q}-\mathbf{q}^{\prime}=(\pi, \pi / 2)$. 
lower is the $T_{c}$. (3) We have studied solutions to a separable pairing potential model in which the susceptibility of Eq. (4c) is replaced by a product of cosine terms: $\cos \left(q_{x} a\right) \cos \left(q_{x}^{\prime} a\right)+\cos \left(q_{y} b\right) \cos \left(q_{y}^{\prime} b\right)$, since it was speculated 16 that this potential would give rise to an 8 node s-state. We find that d-wave states arise naturally in this model as well, and they are always more stable than s-wave states.

FIG. 3. Effect of inter-layer correlation on the superconducting transition temperature $T_{c} .(\triangle)$ indicate the $(d, d)$ states and $(\bigcirc)$ represent the $(s,-s)$ states. We consider different shapes of Fermi surface and Fermi energies - orthorhombic lattice with next-nearest-neighbor hopping, $E_{F}=-0.2$ (dotted line), $E_{F}=-0.1$ (solid line); and tetragonal lattice with $t^{\prime}=0, E_{F}=-0.6$ (dot-dashed line), $E_{F}=-0.4$ (dashed line).

In Fig. 3 is plotted the dependence of the transition temperature on the inter-plane coupling constant $T_{c}$ for the magnetic pairing model of Fig.1 (a), for two different Fermi surface shapest corresponding to $\mathrm{YBaCuO}$ (with two hole concentrations as well $)$ and $\mathrm{LaSrCuO}\left(t^{\prime}=0\right.$ ). Here, because we use a weak coupling approximation, the absolute values of $T_{c}$ are not meaningful. However, the relative changes with different parameterizations are expected to be accurately captured. The circles correspond to the out-of-phase s-states and triangles to in-phase d-states. As expected, for sufficiently small inter-plane coupling the d-wave state is the more stable; however depending on the bandstructure and Fermi surface shape, this state may be readily de-stabilized to a pair of s states by the introduction of a very small interplane interaction. This reflects the general result that (nodeless) s-wave states are able to take better advantage of the superconducting interaction than can d-wave states, which require a cancellation of positive and negative terms to satisfy the gap equation. Note that in the LaSrCuO model the $T_{c}$ 's are generally higher as a consequence the better Fermi surface nesting along the direction of the wave-vector $(\pi, \pi)$. For each parameter set, the various curves tend to coalesce at higher $\lambda_{B}$, where the (s-like) states are found to be more isotropic. This is a consequence of the fact that these isotropic states are not able to utilize the Van Hove singularity effect, which is relatively more important for $\mathrm{d}_{x^{2}-y^{2}}$ state.
In summary, by solving the gap equation for bi-layer models with general repulsive interactions, we find that $\mathrm{d}_{x^{2}-y^{2}}$ states arise quite generally and are not uniquely associated with wave-vector structure along the antiferromagnetic direction. Moreover, we have established that $\pi$ phase shift behavior, which is often cited as the strongest evidence for d-wave pairing can also be associated with (orthorhombic) s-symmetry when the two sub-band gaps have opposite phase. This state has some advantages over d-states in large part because of the relatively small sensitivity of $T_{c}$ to non-magnetic impurities. An important conclusion from our analysis is that there are always competing states in bi-layer systems, and that the order parameter symmetry would be expected to vary from cuprate to cuprate as well as within a given cuprate class at different hole concentrations. One can conclude that the Josephson junction data, in particular, provide strong evidence for superconductivity mediated by some form of repulsive interaction. On the other hand, these collected observations (in bi-layer cuprates) weaken the often cited support for theories of spin fluctuation mediated superconductivity.

We acknowledge useful conversations with A. Leggett, M. Norman and P. Wiegmann, and correspondence with V. Emery. This work was supported by the NSF through the Science and Technology Center for Superconductivity (DMR 91-20000).

${ }^{1}$ D.A. Wollman, D.J. Van Halingen, W.C. Lee, D.M. Ginsberg, and A.J. Leggett, Phys. Rev. Lett. 71, 2134 (1993).

2 J.R. Kirtley, C.C. Tsuei, J.Z. Sun, C.C. Chi, L.S. YuJahnes, A. Gupta, M. Rupp, and M.B. Ketchen, preprint.

${ }^{3}$ A. Mathai, Y. Gim, R.C. Black, A. Amar, and F.C. Wellstood, preprint.

${ }^{4}$ D.A. Brawner and H.R. Ott, Phys. Rev. B 50, 6530 (1994).

${ }^{5}$ A.I. Liechtenstein, I.I. Mazin, and O.K. Andersen, preprint.

${ }^{6}$ N. Bulut, D.J. Scalapino, and R.T. Scalettar, Phys. Rev. B 45, 5577 (1992).

${ }^{7}$ Q. Si, Y. Zha, K. Levin, and J.P. Lu, Phys. Rev. B 479055 (1993).

${ }^{8}$ We do not include any effects of inter-band pairing, which we find are unphysical because they introduce additional transition temperatures.

${ }^{9}$ Y. Zha, Q. Si, and K. Levin, Physica C 212, 413 (1993).

10 J.M. Tranquada, P.M. Gehring, G. Shirane, S. Shamoto, and M. Sato, Phys. Rev. B 46, 5561 (1992); Sternlieb et al, Phys Rev. B 47, 5320 (1993).

${ }^{11}$ K. Levin, Y. Zha, R.J. Radtke, Q. Si, M.R. Norman, and H.B. Schüttler, J. Superconductivity, 7, 563 (1994).

12 Besides those shown in the figures, other s- and d- phases may appear for restricted regions of our parameter space. As a result of the Van Hove effect the $d_{x^{2}-y^{2}}$ form of the d- representation tends to be more robust.

13 A.G. Sun, D.A. Gajewski, M.B. Maple, and R.C. Dynes, 
Phys. Rev. Lett. 72, 2267 (1994).

${ }^{14}$ W.N. Hardy et al, Phys. Rev. Lett. 70, 3999(1993).

${ }^{15} \mathrm{H}$. Ding et al, preprint.

${ }^{16}$ A.J. Fedro and D.D. Koelling, Phys. Rev. B 47, 14342 (1993).

Note Added:

After this manuscript was submitted we received a preprint from K. Kuboki and P. A. Lee, in which an RVB description of bi-layer superconductivity was used to infer a spontaneous breaking of tetragonal symmetry. This $s, d$ mixing will not occur in the present model, as a result of the free energy form which contains a quadratic, rather than quartic, mixing of the in- and out- of- plane gaps. 